\title{
Research of Current Status and the Progress of Digital Oral Implant Therapy Wennuo Chen ${ }^{1, a}$ \\ ${ }^{1}$ Luohe Medical College, Luohe, Henan, 462000 \\ ${ }^{\mathrm{a}}$ email
}

Keywords: Implant; Computer Aided Design And Manufacture; Guide Template

\begin{abstract}
However, this method can only provide two-dimensional planar images, and only indirectly through the two-dimensional image to push the three-dimensional structure of the relationship is the so-called "thinking three-dimensional", with the oral dental implant With the development of computer tomography, physicians can obtain sequence images of the human body structure. However, this inference is very dependent on the physician's experience. In this paper, three-dimensional reconstruction of the oral cavity, positioning and navigation, integrated information and responsibility, surgery-oriented template preoperative simulation and clinical application are summarized below.
\end{abstract}

\section{Introduction}

From the 1960s, Branemark successfully implanted dental implants in the restoration of teeth, and in the early 1980s put forward the theory of bone bonding, bone bonding theory has been widely accepted by domestic and foreign scholars and applied to clinical practice. At present, the international mainstream system of planting 10 years success rate has reached more than $97 \%$. Throughout the history of dental implant development, combined with clinical practice, the development of dental implantation for the following elaboration.

With the development of computer technology, the 3D dental collar model can be easily digitized by intraoral or extraoral measurement technology, which makes the CAD / CAM technology be introduced into the oral repair system, and in clinical application (such as orthodontics, Surgery, etc.) in the digital model of the process, due to oral malformations caused by overlapping interference between the teeth, measuring equipment, precision and low resolution of surface reconstruction methods such as the impact of factors, Adjacent teeth of the model are stuck together without clear teeth, resulting in the loss of localized shapes of the individual teeth. In the dental CAD/CAM system, the inlay, veneer, full crown, partial crown, simple fixed bridge and complete denture are required to be independent of each other and have a single tooth model with original shape. Therefore, it is very important to accurately model the tooth shape of the 3D dental model.

\section{Conventional Oral Implant}

Oral implant is one of the most important methods of oral cavity repair in recent decades. It is compatible with removable denture and the advantages of fixed denture, tooth loss by patients of all ages. Although X-ray tomography can reveal certain information about the bone relationship around the implantation area, this method can only provide two-dimensional planar images. Physicians can only pass through the two-dimensional images To infer the three-dimensional anatomical relationship, the so-called "three-dimensional thinking." With the development of computer tomography technology, physicians can get the human body structure of the sequence image. Through the simple superposition of a series of two-dimensional tomographic image sequences, a richer three-dimensional structure can be deduced based on clinical experience. However, this inference is very dependent on the physician's clinical experience. In order to improve the accuracy and scientificity of medical diagnosis and treatment planning, it is always a hot research topic both at home and abroad to change from two-dimensional fault image sequence to three-dimensional 
image with intuitive three-dimensional effect. This technique is useful for predicting the condition of the alveolar bone, the location of the alveolar nerve, and whether the implant will affect the superior sinus prior to oral implantation

Only to find the patient's oral structure with unexpected abnormalities, then termination of surgery is to reduce surgical trauma. In addition, the operation of the operating bias often can not make the operation can not achieve the desired results, or even failure. The incidence of this phenomenon is increasing the frequency of surgery-oriented template has become another focus of dental implant technology.

\section{Simplant Software System}

The SimPlant system is an interactive computer-aided implantation software developed specifically for oral implantation by Materialise in Belgium, which makes placement of the implant a predictable rather than a guess. The SimPlant system accepts DICOM files derived from commonly used CT scanners for 3D reconstruction; precise navigation between 2D and 3D views; comprehensive information measurement including distance, angle, and BMD; simulation planning for implant surgery and customization Mm-precision surgical guide template, the planning of the perfect surgical program in the clinical simple and accurate implementation.

CT has provided the most comprehensive and accurate image information of the patient's oral structure. SimPlant reconstructs the three-dimensional model from the CT image, which can truly and completely reproduce the patient's anatomical structure in the computer, including the importance of the CT image. Structure is as the collar sinus, inferior alveolar nerve and other parts of the visual display, to build a full range of planting surgery simulation environment. Multi-window display is providing a variety of information at the same time the possibility of reference. The physician may choose to simultaneously display one or more views of the CT sequence, axial, coronal, sagittal, X-ray, and three-dimensional views on the screen. View and view are interrelated to form an accurate real-time positioning navigation relationship. When a position is selected on the 3D model, the cursor will be positioned on the $2 \mathrm{D}$ point in the other view. In combination with rotation, scaling, and transparency, the physician can also select the desired cross-sectional location on the 3D view, hide the front section of the section, and easily view any localized internal bone structures, implants and nerves.

The correct implantation of the implant on the alveolar bone is the key to the successful operation of the implant, and it directly affects the effect and helps to promote the integration of bone tissue. It can accurately measure the bone width, distance between the alveolar nerve and the superior sinus, To determine the placement of the implant space angle, are preoperative should master the important parameters. The density of the bone and the anatomical relationship between the implanted sites determine the degree of deformation around the implant. Implantation is not the time, the axial force generated by the mechanical stress on the surface of the implant can lead to bone around the implant atrophy, which directly affects the success of implant implantation, so a comprehensive understanding of the operation before surgery The structure and density of bone tissue, as well as adjacent anatomical structures, are important for accurate implantation. SimPlant not only provides accurate measurement of regional bone density, but also through a clear display of different colors. In addition, changes in the tissue surrounding the implanted implant were assessed.

SimPlant's implant library is compatible with more than 1,400 implants, including the true shape and size of implants and bridges, to ensure accurate placement in the patient's mouth, so that the design and surgical guide template consistent with the clinical program. The SimPlant software provides regular extensions and updates of the implant library, while the implant library also allows you to add custom implants. The distance between the implant, the distance between the implant and the tooth, and the distance between the implant and the alveolar nerve during the implant procedure are all factors that need to be considered when placing the implant. SimPlant has the function of automatically detecting the interference collision. The software sets a safe space for these distances. When the implant is placed, once the distance is less than the safe value, the system will pop up the alarm prompt to avoid the omission. SimPlant will also alert you when the implant's 
repair space crosses. Scanning, the patient will generally wear scanning dentures, scanning dentures is the ideal effect after the repair. With scanning dentures, physicians more easily determine

Implant placement. When the patient does not wear the scan dentures, SimPlant provides the function of the virtual tooth, properly simulate the restoration of the crown, which is beautiful implant placement. In the CT image, in addition to noise, jitter, etc. will have an impact on imaging, the patient's oral metal objects will also have a casual, affecting the image quality, especially when the serious casual, the imaging results are not ideal, then virtual teeth Can provide a convenient solution. SimPlant not only provides the ability to pick bone at the site of the bone, but also the amount of material required to replenish it, SimPlant calculates the weight of the material required for the bone. SimPlant's 3D view provides intuitive communication tools for the entire treatment team, including physicians, physicians, and patients, making communication clearer and easier for both patients and patients to understand and collaborate.

Power and patient acceptance and satisfaction. In addition, SimPlant provides a variety of regular medical reports for physicians to facilitate archiving and querying.

\section{Surgical Guide Template Application}

At present, although implant surgeons with extensive clinical experience are able to grasp the position, orientation and depth of dental implants in surgical procedures based on the anatomical structure of the edentulous area revealed by conventional panoramic radiographs of bone, it is not uncommon for many complex cases, Is missing in the region of multiple teeth, bone graft defects or bone defects such as the existence of the situation, often easily lead to errors, resulting in surgery alveolar bone perforation, root injury or implant position, direction and depth of the poor And other phenomena.

In the clinical, surgical template as a final information carrier, the dental implant surgery design ideas through the intraoperative template accurate and precise positioning and guidance given to achieve, for the restoration of this complex project safety and functional and aesthetic effects both have important clinical significance. Computer - aided design, computer tomography, stereoscopic imaging technology and patient oral model of multiple technologies in one of the SurguGuide. The accuracy beyond the millimeter, to achieve a few millimeters of ultra-millimeter-level accuracy, combined with SimPlant's surgical design, can get good aesthetic results, to achieve a higher success rate. It makes it possible to pre-fabricate temporary restorations and "instant load". In the real operation process, reduce the occurrence of emergencies, by preventing drill bit deviation, reduce the complexity of surgery to achieve a high degree of accuracy, increase the patient's comfort, so that repair teeth totally natural. According to the different parts of the support, SurgiGuide is divided into three types of bone support, mucous membrane support and dental support.

The bone-supported SurgiGuide rests on the patient's collar bone and is suitable for patients with or without edentulous teeth. Bone-supported SurgiGuide requires the patient's collar bone to have enough bone tissue to ensure that SurgiGuide is placed in a unique and stable position. Manufacturing SurgiGuide requires that the patient's collar can not have a scanning denture. During surgery, the periodontal margins are cut and the bony mucosal wing is opened to separate it from the bone table. Place the SurgiGuide stably on the bone surface and guide the drill into the preoperative position to complete the drilling and reaming. Then place the implant, bring the healing cap, suture mucosal wing.

Mucosal support of the SurgiGuide placed in the soft tissue of the patient's collar, suitable for patients with completely toothless. To create this SurgiGuide, the patient's mucosal surface information needs to be clear. Patients need to carry on the CT scan completely radiopaque scan dentures. So that the designed tooth shape and mucosal profile can be clearly seen on the image. During surgery, a stable and unique position, referred to during the manufacture of the Su-Face Guide, is placed on the soft tissue to guide the drill into the preoperative design position. Using the SurgiGuide mucosal support case to drill the micropores in the mucosa is enough to guide the placement of the implants accurately. This drilling method preserves the vascular structure, 
reducing healing time, it can achieve minimally invasive surgery.

The tooth-supported SurgiGuide rests on the patient's soft tissue and residual teeth. It is suitable for patients with single teeth or small amounts of tooth loss. Dental support is an excellent choice for minimally invasive surgery because all implants are pre-engineered with SimPlant and are fully predictive of bone information without the need to drill and place the implant at the edge of the bone. Just through the mucosa through the micro-hole can guide the precise placement of implants. To manufacture this SurgiGuide, it is necessary to send the implant design and the patient's dental plaster model to Materialise. Materialise uses both types of information to create precise SurgiGuide, which allows it to be precisely and stably placed on the tooth, thus translating the implant design into a real surgical procedure.

\section{The Prospects and Shortcomings}

SimPlant-assisted implant planning and guidewire design make implant placement no longer a traditional guesswork. Reduce the occurrence of intraoperative accidents, improve the success rate of surgery and reduce the operation time. SimPlant's intuitive approach enhances communication between treatment teams, facilitates patient confidence and satisfaction, and enhances collaboration and understanding between physicians and patients. Oral implant planning and guided template design, including three-dimensional image reconstruction and implant planning, registration, real-time navigation and other technology applications. It can provide more scientific and accurate anatomical information for the diagnosis, analysis and measurement of orthopedic bone before operation, reduce the occurrence of intraoperative and postoperative complications, and improve the success rate of operation. Intuitive means of communication is to strengthen the exchange of the treatment team to explore, more likely to build the patient's trust and satisfaction, to enhance the cooperation between doctors and patients and understanding. So SimPlant software can be more accurate design of the entire process of oral implant, more accurate and perfect the general limitations of the full-mouth surface slices and shortcomings, but also better design of dental implant, the technology and methods will become the new research Direction and research topic.

\section{Acknowledgements}

This article is the Henan Science and Technology Department in 2016 basis and the frontier planning project stage results.

Item Number: 162300410267

\section{References}

[1 ] Hajeer MJ, Millett DT, Ayoub AF, et al. Applications of3 D imaging in orthodontics ; Part 1 [ J ].Iorthodon,2004;319(1);62-70

[2 ] Rudolph H, Luthardt RG, water MH. Computer-aided analysis of the influence of digitizing and surfacing on the accuracyin dental CAD, CAM technology [J]. Computer, 2007;37(5):579-587

[3] Liu PR. A panorama of dental CAD/CAM restorative systerns [J].Compend,2005;26(7):507-513

[4] Touchstone A , Phinips RJ. Simplifying CAD/cAM dentistry[OL].(2005-11-09 ) 2009-04-24

[5] Garino F. The orthodontic 3 D world; the basic i01 a of the orthodonti8t [ OL].(2007-08-01)2009-04-24

[ 6 ] Zachow e , Hege HC , Deuflhard P. computer_assisted planring in cranj-maxillofacial surgery [J] . Comput Inform Technology, 2006, 14 (1); 53-64 